\title{
Successful Aging in Individuals From Less Advantaged, Marginalized, and Stigmatized Backgrounds
}

\author{
Myriam V. Thoma ${ }^{\mathrm{ab}}$, Shauna L. Mc Gee ${ }^{\mathrm{ab}}$ \\ [a] Psychopathology and Clinical Intervention, Institute of Psychology, University of Zurich, Zurich, Switzerland. \\ [b] University Research Priority Program “Dynamics of Healthy Aging”, University of Zurich, Zurich, Switzerland.
}

Clinical Psychology in Europe, 2019, Vol. 1(3), Article 32578, https://doi.org/10.32872/cpe.v1i3.32578

Received: 2018-12-20 • Accepted: 2019-03-27 • Published (VoR): 2019-09-20

Handling Editor: Cornelia Weise, Philipps-University of Marburg, Marburg, Germany

Corresponding Author: Myriam V. Thoma, Department of Psychology, University of Zurich, Binzmühlestrasse 14/17, 8050 Zurich, Switzerland. Tel.: +41 63573 06. E-mail: m.thoma@psychologie.uzh.ch

\begin{abstract}
Background: Health and well-being in later life are heavily influenced by behaviors across the life course, which in turn are influenced by a variety of wider contextual, social, economic, and organizational factors. There is considerable potential for inequalities in health-promoting behaviors and health outcomes, arising from poverty, social, and environmental factors. This suggests that individuals from disadvantaged backgrounds and circumstances may have more exposure to (chronic) stressors, coupled with reduced access to resources, and increased susceptibility to risk factors for ill-health and mental disorders in later life. This drastically decreases the likelihood for successful aging in individuals from less advantaged backgrounds. Nevertheless, despite these adverse circumstances, some high-risk, disadvantaged individuals have been shown to achieve and maintain good health and well-being into later life.

Method: This scientific update provides an overview of recently published research with samples that, against expectations, demonstrate successful aging.

Results: Favorable personality traits, cognitive strategies, and a high-level of intrinsic motivation, paired with a supportive social environment, have been found to build a prosperous basis for successful aging and positive health outcomes in later life for individuals living in aversive environmental circumstances.

Conclusion: For clinical psychologists, the movement towards the investigation of underlying mechanisms of successful aging from a psychological perspective, particularly in disadvantaged individuals, may be a critical step towards understanding the vast heterogeneity in aging.
\end{abstract}

\section{Keywords}

successful aging, disadvantaged backgrounds, marginalization and stigmatization, LGBT, disparities in racial and ethnic minorities 


\section{Highlights}

- Successful aging is possible in disadvantaged individuals.

- Psychological and social resilience resources may compensate for the impact of disadvantage.

- The application of multi-level resilience models can aid future research on successful aging.

Old age is a life stage characterized by a high degree of diversity between individuals. A growing body of literature has been dedicated to understanding this heterogeneity in aging. Special focus has been placed on the positive end of the aging spectrum. At the moment, there exists no universally-accepted definition for what constitutes this "positive end of the aging spectrum". The "successful aging" (SA) construct, which is often used in research to examine positive aging research questions, is currently defined and operationalized in more than 100 different ways (Cosco, Prina, Perales, Stephan, \& Brayne, 2014). However, despite the current lack of a commonly-accepted definition, experts in the field generally agree that the SA construct should consist of several different dimensions, including a (mental and physiological) health facet, a (subjective) well-being facet, as well as a social (engagement) facet (Kleineidam et al., 2018). Nevertheless, despite its broad variety of operationalizations, the SA construct as a whole constitutes a meaningful and useful construct that can be applied to examine why some individuals are more likely than others to remain predominantly healthy and maintain a high level of physical functionality and social activity even into older age (Kleineidam et al., 2018).

In this regard, it is particularly useful to examine what factors can be identified in connection with more favorable aging processes and outcomes. Previous research on SA has uncovered a range of predictors, including socio-demographic factors and specific behaviors linked to SA. Socio-economic status (SES; including income and wealth), education, and health-promoting behaviors (e.g., non-smoking, healthy diet, physical activity), are among the frequently identified predictors for SA (e.g., Daskalopoulou et al., 2018; Gopinath, Kifley, Flood, \& Mitchell, 2018; Kok, Aartsen, Deeg, \& Huisman, 2016; Vauzour et al., 2017; Whitley, Benzeval, \& Popham, 2018). Other branches of health-related research can also provide additional evidence for potential risk and protective factors that are essential in predicting SA. For instance, an important line of research on the (longterm) impact of early-life stress suggests that childhood neglect and abuse can increase the risk of future ill health and mental disorders, and may thus diminish the probability of SA (Jones, Nurius, Song, \& Fleming, 2018; Nurius, Fleming, \& Brindle, 2017). In fact, supporting this, a recently published large-scale longitudinal study found a meaningful link between early-life stress and SA trajectories (Kok, Aartsen, Deeg, \& Huisman, 2017).

Upon closer examination of the factors that may promote or hinder SA, it appears as if SA may be a rather elitist paradigm, seemingly reserved for more advantaged and al- 
ready healthy individuals. That is, those who grow up in more functional families, who were provided with the opportunity for a good education, and the necessary access to health-literacy and services, coupled with the essential assets and time required to implement health-promoting behaviors. This leaves a rather pessimistic prospect for individuals who grew up and lived in poverty or come from underprivileged educational backgrounds; as well as for those who experienced early misfortune in the form of childhood stressful life events, abuse and neglect, or those living with chronic health conditions (e.g., Bøe, Serlachius, Sivertsen, Petrie, \& Hysing, 2018; Kok et al., 2017; Lê-Scherban et al., 2018). Are these individuals deprived of the opportunity to age successfully?

This scientific update aims to answer this question by providing an overview of the latest research dedicated to the investigation of SA in individuals from less advantaged backgrounds. For this, a list of 'disadvantaged' groups was first compiled and the search was then limited to articles on older adults. A search of the databases was conducted for articles published since 2017. Given the wide diversity in how the SA construct is currently defined in the scientific literature, the search was not restricted by applying a particular SA definition or operationalization.

\section{Successful Aging Within the Context of Socio- Economic Disadvantage and Childhood Adversity}

The research group of Kok and colleagues (Kok, van Nes, Deeg, Widdershoven, \& Huisman, 2018) qualitatively examined SA in Dutch individuals $\left(N=11\right.$; Age $_{\text {range }}=78-93$ years) who had a low lifetime socio-economic position (SEP). More specifically, the authors were interested in the identification of resilience factors that protected those individuals from the potentially negative impact of chronic socio-economic adversity. Several resilience-enhancing factors were identified, including 'social support', 'generativity', 'pro-active management', 'cognitive restructuring', 'enduring', and 'surrendering'. Confirming, but also expanding the resilience conceptualization for low SEP individuals previously proposed by Chen and Miller (2012), the authors concluded that in addition to mental re-evaluation of the disadvantaged background, it also appears to be necessary to have a supportive social environment (which also requires pro-social behaviors) and the will to actively confront the external circumstances in question, i.e., by changing the adverse environment with one's own actions (Kok et al., 2018).

Another recent study (Scelzo et al., 2018) examined a sample of very old individuals living in rural villages in the southern part of Italy $\left(N=29\right.$; Age $_{\text {range }}=90-101$ years $)$. Rural areas tend to show health-disparities due to a disproportionate lack of services in medically underserved areas, issues of access to existing services, differences in patient expectations and health-seeking behaviors, as well as the delivery of health care (Douthit, Kiv, Dwolatzky, \& Biswas, 2015). While not directly assessing SA in this study, 
(extreme) longevity can be regarded (and has previously been used) as a proxy to assess (the consequences of) SA (see Cosco et al., 2014). The qualitative part of this mixed-method study examined themes related to (extreme) longevity. Common themes associated with SA were identified, including 'positivity', i.e., resilience and an optimistic outlook on life (also including self-efficacy and perseverance); being a 'controlling' or 'strong' personality; being socially active and engaged, i.e., having tight bonds with family members; religious beliefs; as well as being hard working; and having a "love of the land" (p. 33). Altogether, the above findings show some parallels to those reported by Kok et al. (2018): In addition to the importance of having mental resources, such as the capacity for acceptance/perseverance (facilitated by religious beliefs or particular personality traits), there is also the necessity of actively engaging with or changing the external environment (e.g., by being hard working), which is framed within the context of a functional and close social network.

Another example of SA despite adverse life circumstances can be seen in the case of Swiss former indentured child laborers (i.e., former Verdingkinder). Due to extreme poverty, death of a parent, divorce, or single motherhood, children were taken away from their parents and placed in foster families (mostly farmers), where they had to work for their living (Leuenberger \& Seglias, 2008). Given that, in those times, the foster-care system was still poorly controlled, these children (in most cases forcefully separated from their families of origin) often experienced little to no protection. Documented by individuals who came forward publicly with their experiences, and corroborated by contemporary witnesses, these biographies are filled with reports of (extreme levels of) childhood abuse and neglect. A qualitative study (Höltge, Mc Gee, Maercker, \& Thoma, 2018a) investigated SA in former Verdingkinder $\left(N=12 ; M_{\text {age }}=71\right.$ years; Age $\left._{\text {range }}=59-88\right)$. SA was defined as (self-rated) good health, feelings of happiness, balance and/or calmness most of the time, and a high level of satisfaction with (social) life. The factors 'light-heartedness', 'social-purpose', and 'self-enhancement' were identified as predictors for SA. These individuals, after what they had endured in early-life, took on a positive perspective following conscious reflection (i.e., a proxy for a resilience resource). While striving to experience positive feelings, they nevertheless kept a realistic perspective on life by acknowledging that negative experiences are part of a normal existence (i.e., cognitive reevaluation). In general, participants could be classified as stress-resilient, a resource that was described to be developed through active coping and by coming to terms with their difficult past. They strongly valued (harmonic) social relationships and had the altruistic desire to help others (i.e., social component). Furthermore, they developed a strong motivation for self-improvement that pushed them to work hard and to continuously engage in further education (i.e., active engagement with external environment). 


\section{Successful Aging in Racial and Ethnic Minorities}

Similar to individuals from disadvantaged backgrounds or adverse childhood circumstances, individuals from minority groups often face issues of marginalization and stigma, which can influence their health and well-being into later life. One such example can be seen in racial and ethnic minorities, who, in addition to potentially higher levels of disadvantages and inequalities (Zubair \& Norris, 2015), can also face problems with exclusion and discrimination, which may compound health issues and ultimately hinder SA (Ferraro, Kemp, \& Williams, 2017). However, despite these additional stressors, evidence is emerging that some individuals can experience good health into older age. For example, a study of perceived discrimination and psychological well-being in African American older adults $\left(N=397\right.$; Age $\left._{\text {range }}=65-89\right)$ found that the characteristic of 'self-acceptance', an awareness and acceptance of personal strengths and weaknesses, was shown to buffer the negative effect of discrimination on depressive symptomology, an indicator of psychological well-being (Yoon, Coburn, \& Spence, 2019). Another study (Klokgieters, van Tilburg, Deeg, \& Huisman, 2018a) examined the potential buffering effect of various religious activities against the negative impact of disadvantage (e.g., no/low resources) in older Turkish and Moroccan immigrants $\left(N=455\right.$; $_{\text {Age }}$ range $=55-66$ years $)$. While a positive relationship was found between well-being and private religious activities, there was no indication of a buffering effect for any of the religious activities against the experienced disadvantage.

\section{Successful Aging in Individuals Living With HIV}

Another minority group that has experienced much stigma and discrimination is that of individuals living with the human immunodeficiency virus (HIV). HIV is a chronic illness, associated with a higher risk of experiencing psychosocial challenges and physiological issues. However, with advances in medicine, individuals with HIV are living longer, better lives and research has started focusing on SA and SA-related factors in this population. A qualitative study in individuals with $\operatorname{HIV}\left(N=30\right.$; Age $\left._{\text {range }}=50-73\right)$ assessed barriers to and strategies for SA (Emlet, Harris, Furlotte, Brennan, \& Pierpaoli, 2017). Results showed that while stigma, prejudice, and discrimination were identified as potential SA barriers, a number of SA-related themes emerged, including resilience components, such as self-care, mastery, and spirituality; social support; and the importance of the environmental context, such as structural support, social networks, and communities. Another qualitative study on SA in individuals with HIV $\left(N=24\right.$; Age $\left._{\text {range }}=50-73\right)$ identified similar themes, emphasizing components over which persons had individual control (Solomon et al., 2018). These included staying positive, maintaining social support and connectedness with others, taking responsibility and being self-reliant for one's well-being, and engaging in meaningful activities. These findings indicate that a combination of 
individual characteristics, such as control and mastery, and external influences, such as social and structural support, are important in fostering SA in this marginalized population.

\section{Successful Aging in LGBT Older Adults}

A population that also faces additional barriers to SA, are lesbian, gay, bisexual, and transgender (LGBT) older adults. Despite the risks of ill-health and social isolation for LGBT older adults (Wright et al., 2017), discrimination is often reported as a barrier to the utilization of health and community services (Alencar Albuquerque et al., 2016). A large-scale study of $N=2,415$ LGBT older adults identified risk and resilience pathways to positive health outcomes in later life (Fredriksen-Goldsen, Kim, Bryan, Shiu, \& Emlet, 2017). Results showed that marginalization was a risk factor associated with fewer social resources and poorer mental health outcomes. However, resilient pathways were identified in which psychological (e.g., positive identity appraisal) and social (e.g., social connectedness) resources were associated with health-promoting behaviors, which in turn facilitated good physical health into older age. These findings suggest that the interaction of social and psychological factors can help LGBT older adults to maintain good health and foster SA, even within an environmental context of marginalization.

The above research suggests that $\mathrm{SA}$ is possible for individuals from less advantaged, marginalized, and stigmatized backgrounds. However, as this is a relatively new and emerging topic, the exact mechanisms through which SA is fostered in these disadvantaged populations are not yet known. Some theories and models are presented in the next section, which may be applied to help explain the underlying mechanisms of this process of SA.

\section{Underlying Mechanisms of Successful Aging}

The 'steeling effect' is one theory that may explain positive health in the face of adversity (Liu, 2015; Rutter, 2006, 2012). This theory proposes that previous exposure to adversity (e.g., disadvantaged circumstances, discrimination) may have a 'steeling' or strengthening effect on individuals, which can increase their resistance to later stress or adversity. It further suggests that moderate adversity may be more beneficial than no or high adversity, as it is adequately challenging to facilitate the development of coping skills and the utilization of resources (for a review see Höltge, Mc Gee, Maercker, \& Thoma, 2018b). However, there is a lack of research applying this model in human studies (for one such study see Höltge, Mc Gee, \& Thoma, 2018), particularly with older adults, and the role of steeling for SA remains poorly understood. Furthermore, while some studies have examined aspects of adverse circumstances as part of a larger assessment, such as exposure to 
social and environmental stress (Seery, Holman, \& Silver, 2010), future research is needed to apply this steeling model specifically to individuals from disadvantaged populations.

The above literature suggests that a combination of psychological and individual factors (e.g., mastery and control, self-efficacy) and external social factors (e.g., social engagement, connectedness with others) can contribute to resilience and SA in disadvantaged populations. However, it is also important to consider the enabling environmental and context factors, which are particularly important in this specific population due to their adverse backgrounds and circumstances. Supporting this is the social ecological model of resilience, which emphasizes the role of environmental factors in health and well-being, and suggests that resilience is facilitated by the interaction between the individual and their environment (Ungar, 2012). Specifically, this model suggests that the environment can facilitate access to resilience-promoting resources; that resilience may differ as a result of the complex and changing nature of an individual's circumstances and the interaction with their personal traits; and that the resilience process is culturally and temporally embedded and is therefore influenced by the cultural norms of the time, which is particularly important for specific cultural groups such as minorities (Ungar, 2011). The importance of the individual-environment interaction for the well-being of disadvantaged populations can be seen in the reoccurring finding that environmental and contextual factors, such as social support and social engagement, were significant in supporting individual resilience and SA (e.g., Emlet et al., 2017; Kok et al., 2018; Scelzo et al., 2018).

Further support comes from a resilience conceptualization in the field of sociology (Schafer, Shippee, \& Ferraro, 2009). Schafer and colleagues (2009) argue that in order to actively buffer or overcome disadvantage, several processes must take place: First, an individual must become aware (i.e., recognition/subjective evaluation) of one's undesirable position, adversity, or misfortune. Second, the individual must take action (i.e., constructive adaptation) to counteract or amend the adverse situation and/or to avoid negative consequences. Third, to efficaciously face disadvantage, one must activate and apply adequate and effective resources. These resources may be located within oneself (e.g., IQ, resiliency traits) and/or within the socio-economic system, in the form of social relationships, SES, and economic resources (Schafer et al., 2009). As in the social ecological model of resilience, this conceptualization highlights the importance of both individual and social-context factors in overcoming disadvantage.

The interaction between individual and environment is also reflected in and supported by another model of resilience by Liu, Reed, and Girard (2017). This model proposes that resilience is dynamic and is formed through the interaction of factors across multiple, interconnected levels. These levels include core resilience, which are inherent, stable characteristics and traits, such as gender, race, and ethnicity; internal resilience, which are learnable and changeable factors, such as active coping, mastery, and self-acceptance; and external resilience, which are contextual and environmental factors, such as social 
resources and support. This model could be applied in future research to better understand the multi-level mechanisms underpinning the processes of resilience and SA.

In addition, the construct 'Sense of Coherence-Revised' (SOC-R) could be examined in conjunction with Liu et al.'s (2017) model. This construct may help explain how individuals can utilize these multi-level factors to facilitate resilience and SA, as it is the ability to integrate and balance positive and negative experiences in order to maintain and develop health and well-being following stress or adversity (Bachem \& Maercker, 2016; Mc Gee, Höltge, Maercker, \& Thoma, 2018a, 2018b). It is based on the salutogenic theory, which views health as a continuum, and proposes that SOC-R can help individuals to utilize resources (e.g., personality traits, mastery, social support) appropriate for their current circumstances and move them towards good health on this continuum (Antonovsky, 1987). In this way, SOC-R can positively influence the aging process and foster SA (Bachem \& Maercker, 2016). The above theories and models provide a theoretical basis for future research to examine the mechanisms and factors associated with resilience and SA, particularly in populations with disadvantaged backgrounds.

It should be emphasized that this article constitutes a short, current update and overview of the latest developments and publications in this particular field. As such, a systematic review of the literature was not conducted. This may have resulted in a non-comprehensive or even biased delineation of existing literature and the deduction of oversimplified conclusions. It is possible that additional mechanisms and factors exist, which were not discussed in this short update that links SA to adverse experiences and disadvantaged backgrounds. The association between SA and disadvantage is complex and underlying mechanisms are still poorly understood. Disadvantage can have multiple forms and can also hide behind alleged "advantaged" circumstances. For instance, a large-scale longitudinal study on SA (Kok et al., 2017) showed that not only low, but also high SEP, was linked to stressful life events (e.g., higher divorce rate). It is also important to note that the potential buffering impact of psychological resources may depend on the particular context, such as the cultural background (Klokgieters, van Tilburg, Deeg, \& Huisman, 2018b).

\section{Conclusion}

Research on SA is continuously uncovering predictors for more favorable aging processes and outcomes. This scientific update and overview focused on very recent developments and trends in this area, examining SA in less advantaged populations. Findings from these studies highlight the importance of considering a combination of psychological and individual resilience factors, as well as external social and environmental components. Individual, psychological, and social factors can play a compensatory role for individuals living with negative environmental influences. For clinical psychologists, the movement towards the investigation of underlying mechanisms of SA from a psychologi- 
cal perspective, particularly in disadvantaged individuals, may be a critical step towards understanding the vast heterogeneity in aging.

Funding: This work was supported by the Swiss Government Excellence Scholarship (ESKAS-Nr. 2016.0109) which funded SLMG's position.

Competing Interests: The authors have declared that no competing interests exist.

Acknowledgments: During the work on her dissertation, Shauna L. Mc Gee was a pre-doctoral fellow of LIFE (International Max Planck Research School on the Life Course; participating institutions: MPI for Human Development, Humboldt-Universität zu Berlin, Freie Universität Berlin, University of Michigan, University of Virginia, University of Zurich).

Author Contributions: MVT - conceptualization, literature research, writing original draft, review and editing of manuscript. SMG - conceptualization, literature research, writing original draft, review and editing of manuscript.

\section{References}

Alencar Albuquerque, G. A., de Lima Garcia, C., da Silva Quirino, G., Alves, M. J. H., Belém, J. M., dos Santos Figueiredo, F. W., ... Valenti, V. E. (2016). Access to health services by lesbian, gay, bisexual, and transgender persons: Systematic literature review. BMC International Health and Human Rights, 16(1), Article 2. https://doi.org/10.1186/s12914-015-0072-9

Antonovsky, A. (1987). Unraveling the mystery of health: How people manage stress and stay well. San Francisco, CA, USA: Jossey-Bass.

Bachem, R., \& Maercker, A. (2016). Development and psychometric evaluation of a revised sense of coherence scale. European fournal of Psychological Assessment, 34, 206-215.

https://doi.org/10.1027/1015-5759/a000323

Bøe, T., Serlachius, A. S., Sivertsen, B., Petrie, K. J., \& Hysing, M. (2018). Cumulative effects of negative life events and family stress on children's mental health: The Bergen Child Study. Social Psychiatry and Psychiatric Epidemiology, 53(1), 1-9. https://doi.org/10.1007/s00127-017-1451-4

Chen, E., \& Miller, G. E. (2012). “Shift-and-persist” strategies: Why low socioeconomic status isn't always bad for health. Perspectives on Psychological Science, 7(2), 135-158. https://doi.org/10.1177/1745691612436694

Cosco, T. D., Prina, A. M., Perales, J., Stephan, B. C., \& Brayne, C. (2014). Operational definitions of successful aging: A systematic review. International Psychogeriatrics, 26(3), 373-381. https://doi.org/10.1017/S1041610213002287

Daskalopoulou, C., Stubbs, B., Kralj, C., Koukounari, A., Prince, M., \& Prina, A. M. (2018). Associations of smoking and alcohol consumption with healthy ageing: A systematic review and meta-analysis of longitudinal studies. BMJ Open, 8(4), Article e019540. https://doi.org/10.1136/bmjopen-2017-019540 
Douthit, N., Kiv, S., Dwolatzky, T., \& Biswas, S. (2015). Exposing some important barriers to health care access in the rural USA. Public Health, 129(6), 611-620. https://doi.org/10.1016/j.puhe.2015.04.001

Emlet, C. A., Harris, L., Furlotte, C., Brennan, D. J., \& Pierpaoli, C. M. (2017). 'I'm happy in my life now, I'm a positive person': Approaches to successful ageing in older adults living with HIV in Ontario, Canada. Ageing \& Society, 37(10), 2128-2151. https://doi.org/10.1017/S0144686X16000878

Ferraro, K. F., Kemp, B. R., \& Williams, M. M. (2017). Diverse aging and health inequality by race and ethnicity. Innovation in Aging, 1(1), Article igx002. https://doi.org/10.1093/geroni/igx002

Fredriksen-Goldsen, K. I., Kim, H., Bryan, A. E. B., Shiu, C., \& Emlet, C. A. (2017) The cascading effects of marginalization and pathways of resilience in attaining good health among LGBT older adults. The Gerontologist, 57(Suppl_1), S72-S83. https://doi.org/10.1038/s41598-018-28526-3

Gopinath, B., Kifley, A., Flood, V. M., \& Mitchell, P. (2018). Physical activity as a determinant of successful aging over ten years. Scientific Reports, 8(1), Article 10522. https://doi.org/10.1038/s41598-018-28526-3

Höltge, J., Mc Gee, S. L., Maercker, A., \& Thoma, M. V. (2018a). Childhood adversities and thriving skills: Sample case of older Swiss former indentured child laborers. American fournal of Geriatric Psychiatry, 26, 886-895. https://doi.org/10.1016/j.jagp.2018.02.002

Höltge, J., Mc Gee, S. L., Maercker, A., \& Thoma, M. V. (2018b). A salutogenic perspective on adverse experiences. European fournal of Health Psychology, 25(2), 53-69.

https://doi.org/10.1027/2512-8442/a000011

Höltge, J., Mc Gee, S. L., \& Thoma, M. V. (2018). The curvilinear relationship of early-life adversity and successful aging: The mediating role of mental health. Aging and Mental Health. Advance online publication. https://doi.org/10.1080/13607863.2018.1433635

Jones, T. M., Nurius, P., Song, C., \& Fleming, C. M. (2018). Modeling life course pathways from adverse childhood experiences to adult mental health. Child Abuse \& Neglect, 80, 32-40. https://doi.org/10.1016/j.chiabu.2018.03.005

Kleineidam, L., Thoma, M. V., Maercker, A., Bickel, H., Mösch, E., Hajek, A., . . Luck, T. (2018). What is successful aging? A psychometric validation study of different construct definitions. Gerontologist. Advance online publication. https://doi.org/10.1093/geront/gny083

Klokgieters, S. S., van Tilburg, T. G., Deeg, D. J., \& Huisman, M. (2018a). Do religious activities among young-old immigrants act as a buffer against the effect of a lack of resources on wellbeing? Aging \& Mental Health. Advance online publication. https://doi.org/10.1080/13607863.2018.1430739

Klokgieters, S. S., van Tilburg, T. G., Deeg, D. J., \& Huisman, M. (2018b). Resilience in the Disabling effect of gait speed among older Turkish and Moroccan immigrants and Native Dutch. fournal of Aging and Health, 30(5), 711-737. https://doi.org/10.1177/0898264316689324

Kok, A. A., Aartsen, M. J., Deeg, D. J., \& Huisman, M. (2016). Socioeconomic inequalities in a 16year longitudinal measurement of successful ageing. Journal of Epidemiology \& Community Health, 70(11), 1106-1113. https://doi.org/10.1136/jech-2015-206938 
Kok, A. A., Aartsen, M. J., Deeg, D. J., \& Huisman, M. (2017). The effects of life events and socioeconomic position in childhood and adulthood on successful aging. Fournals of Gerontology: Series B, 72(2), 268-278. https://doi.org/10.1093/geronb/gbw111

Kok, A. A. L., van Nes, F., Deeg, D. J. H., Widdershoven, G., \& Huisman, M. (2018). "Tough times have become good times": Resilience in older adults with a low socioeconomic position. Gerontologist, 58(5), 843-852. https://doi.org/10.1093/geront/gny007

Lê-Scherban, F., Brenner, A. B., Hicken, M. T., Needham, B. L., Seeman, T., Sloan, R. P., . . Roux, A. V. D. (2018). Child and adult socioeconomic status and the cortisol response to acute stress: Evidence from the multi-ethnic study of atherosclerosis. Psychosomatic Medicine, 80(2), 184-192. https://doi.org/10.1097/PSY.0000000000000543

Leuenberger, M., \& Seglias, L. (2008). Versorgt und vergessen. Ehemalige Verdingkinder erzählen. Zürich, Switzerland: Rotpunktverlag.

Liu, J. J., Reed, M., \& Girard, T. A. (2017). Advancing resilience: An integrative, multi-system model of resilience. Personality and Individual Differences, 111, 111-118. https://doi.org/10.1016/j.paid.2017.02.007

Liu, R. T. (2015). A developmentally informed perspective on the relation between stress and psychopathology: When the problem with stress is that there is not enough. fournal of Abnormal Psychology, 124(1), 80-92. https://doi.org/10.1037/abn0000043

Mc Gee, S. L., Höltge, J., Maercker, A., \& Thoma, M. V. (2018a). Evaluation of the revised Sense of Coherence scale in a sample of older adults: A means to assess resilience aspects. Aging \& Mental Health, 22, 1438-1447. https://doi.org/10.1080/13607863.2017.1364348

Mc Gee, S. L., Höltge, J., Maercker, A., \& Thoma, M. V. (2018b). Sense of coherence and stressrelated resilience: Investigating the mediating and moderating mechanisms in the development of resilience following stress or adversity. Frontiers in Psychiatry, 9, Article 378. https://doi.org/10.3389/fpsyt.2018.00378

Nurius, P. S., Fleming, C. M., \& Brindle, E. (2017). Life course pathways from adverse childhood experiences to adult physical health: A structural equation model. Journal of Aging and Health, 31(2), 211-230. https://doi.org/10.1177/0898264317726448

Rutter, M. (2006). Implications of resilience concepts for scientific understanding. Annals of the New York Academy of Sciences, 1094(1), 1-12. https://doi.org/10.1196/annals.1376.002

Rutter, M. (2012). Resilience as a dynamic concept. Development and Psychopathology, 24(2), 335-344. https://doi.org/10.1017/S0954579412000028

Scelzo, A., Di Somma, S., Antonini, P., Montross, L. P., Schork, N., Brenner, D., \& Jeste, D. V. (2018). Mixed-methods quantitative-qualitative study of 29 nonagenarians and centenarians in rural Southern Italy: Focus on positive psychological traits. International Psychogeriatrics, 30(1), 31-38. https://doi.org/10.1017/S1041610217002721

Schafer, M. H., Shippee, T. P., \& Ferraro, K. F. (2009). When does disadvantage not accumulate? Toward a sociological conceptualization of resilience. Swiss fournal of Sociology, 35(2), 231-252. 
Seery, M. D., Holman, E. A., \& Silver, R. C. (2010). Whatever does not kill us: Cumulative lifetime adversity, vulnerability, and resilience. Journal of Personality and Social Psychology, 99(6), 1025-1041. https://doi.org/10.1037/a0021344

Solomon, P., Letts, L., O'Brien, K. K., Nixon, S., Baxter, L., \& Gervais, N. (2018). 'I'm still here, I'm still alive': Understanding successful aging in the context of HIV. International fournal of STD \& AIDS, 29(2), 172-177. https://doi.org/10.1177/0956462417721439

Ungar, M. (2011). The social ecology of resilience: Addressing contextual and cultural ambiguity of a nascent construct. American fournal of Orthopsychiatry, 81(1), 1-17.

https://doi.org/10.1111/j.1939-0025.2010.01067.x

Ungar, M. (2012). Researching and theorizing resilience across cultures and contexts. Preventive Medicine, 55(5), 387-389. https://doi.org/10.1016/j.ypmed.2012.07.021

Vauzour, D., Camprubi-Robles, M., Miquel-Kergoat, S., Andres-Lacueva, C., Bánáti, D., BarbergerGateau, P., . . Hogervorst, E. (2017). Nutrition for the ageing brain: Towards evidence for an optimal diet. Ageing Research Reviews, 35, 222-240. https://doi.org/10.1016/j.arr.2016.09.010

Whitley, E., Benzeval, M., \& Popham, F. (2018). Associations of successful aging with socioeconomic position across the life-course: The West of Scotland Twenty-07 Prospective Cohort Study. Fournal of Aging and Health, 30(1), 52-74.

https://doi.org/10.1177/0898264316665208

Wright, L. A., King, D. K., Retrum, J. H., Helander, K., Wilkins, S., Boggs, J. M., . . Gozansky, W. S. (2017). Lessons learned from community-based participatory research: Establishing a partnership to support lesbian, gay, bisexual and transgender ageing in place. Family Practice, 34(3), 330-335. https://doi.org/10.1093/fampra/cmx005

Yoon, E., Coburn, C., \& Spence, S. A. (2019). Perceived discrimination and mental health among older African Americans: The role of psychological well-being. Aging \& Mental Health, 23, 461-469. https://doi.org/10.1080/13607863.2017.1423034

Zubair, M., \& Norris, M. (2015). Perspectives on ageing, later life and ethnicity: Ageing research in ethnic minority contexts. Ageing \& Society, 35(5), 897-916.

https://doi.org/10.1017/S0144686X14001536

\section{EACLIPT}

Clinical Psychology in Europe (CPE) is the official journal of the European Association of Clinical Psychology and Psychological Treatment (EACLIPT).

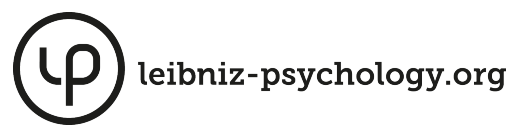

PsychOpen GOLD is a publishing service by Leibniz Institute for Psychology Information (ZPID), Germany. 